Originalien

Anaesthesist $2021 \cdot 70: 213-222$

https://doi.org/10.1007/s00101-020-00874-8

Eingegangen: 7. März 2020

Überarbeitet: 21. Juli 2020

Angenommen: 25. Juli 2020

Online publiziert: 26 . Oktober 2020

() Der/die Autor(en) 2020

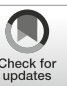

J. Spielberger ${ }^{1} \cdot$ F. Heid ${ }^{1} \cdot$ I. Schmidtmann ${ }^{2} \cdot$ P. Drees ${ }^{3} \cdot$ U. Betz ${ }^{4} \cdot$ W. Schwaderlapp ${ }^{1} \cdot$

\title{
G. Pestel
}

' Klinik für Anästhesiologie, Universitätsmedizin Mainz, Mainz, Deutschland

${ }^{2}$ Institut für Medizinische Biometrie, Epidemiologie und Informatik, Universitätsmedizin Mainz, Mainz, Deutschland

${ }^{3}$ Zentrum für Orthopädie und Unfallchirurgie, Universitätsmedizin Mainz, Mainz, Deutschland

${ }^{4}$ Institut für Physikalische Therapie, Prävention und Rehabilitation, Universitätsmedizin Mainz, Mainz,

Deutschland

\section{Patient*innenzentrierte} perioperative Versorgung: perioperative Prozessqualität, Effektivität von Schmerztherapie und Mobilisationsfortschritt nach Implementation eines Maßnahmenbündels bei Knietotalendoprothese

Diagnostik, Therapie und Nachsorge im Rahmen operativer Eingriffe wurde in den USA das Konzept des "perioperative surgical home" [1] initialisiert. Dieses Konzept basiert auf der Vorstellung, dass die lange Zeit übliche vertikale Strukturierung des Gesundheitswesens in ein teamorientiertes Vorgehen transformiert werden sollte [15], in dem bei patientenspezifischer Anwendung von „evidencebased medicine" ein umfassender und zügiger Heilverlauf angestrebt wird, zum Wohle der Patient ${ }^{*}$ innen, der Leistungserbringer und der Gesellschaft [28]. Zentraler Leitgedanke des Konzepts ist die interdisziplinäre Kooperation aller beteiligten Berufsgruppen mit dem Ziel einer optimalen Patientenversorgung bei umsichtiger Nutzung von Zeit und Ressourcen [5]. Dabei arbeitet ärztliches und nichtärztliches Personal gemeinsam an der Aufgabe, für die jeweilige Institution ein Konzept zu erarbeiten, in dem informierte und aufgeklärte Patient ${ }^{*}$ innen nach Regeln gesicherten Wissens und guter klinischer Praxis mittels ineinan- dergreifender Behandlungsschritte von der präoperativen Entscheidungsfindung bis zur postoperativen Nachbetreuung kontinuierlich anhand konsensual entwickelter Schulungs- und Behandlungsprotokolle versorgt werden [15, 27].

$\mathrm{Da}$ im deutschen Gesundheitswesen ein solches Vorgehen bislang nicht evaluiert ist, werden mit vorliegender Studie Aspekte der perioperativen Prozessqualität am Beispiel der Anästhesieeinleitungszeit, der suffizienten Schmerztherapie und des zügigen Mobilisationsfortschrittes vor und nach Implementation eines Maßnahmenbündels an der Universitätsmedizin Mainz (präoperative $\mathrm{Pa}$ -

\section{Infobox 1 „matched-pair"-} Kriterien

1. Alterskohorte (Fünfjahreszeitraum)

2. Geschlecht männlich vs. Geschlecht weiblich

3. $\mathrm{BMI}<25 \mathrm{~kg} / \mathrm{m}^{2}$ vs. BMI $25-30 \mathrm{~kg} / \mathrm{m}^{2}$ vs. $\mathrm{BMI}>30 \mathrm{~kg} / \mathrm{m}^{2}$

4. ASA-PS 1 und 2 vs. ASA-PS 3
Die Untersuchung ist Teil der Dissertation von Frau W. Schwaderlapp. 


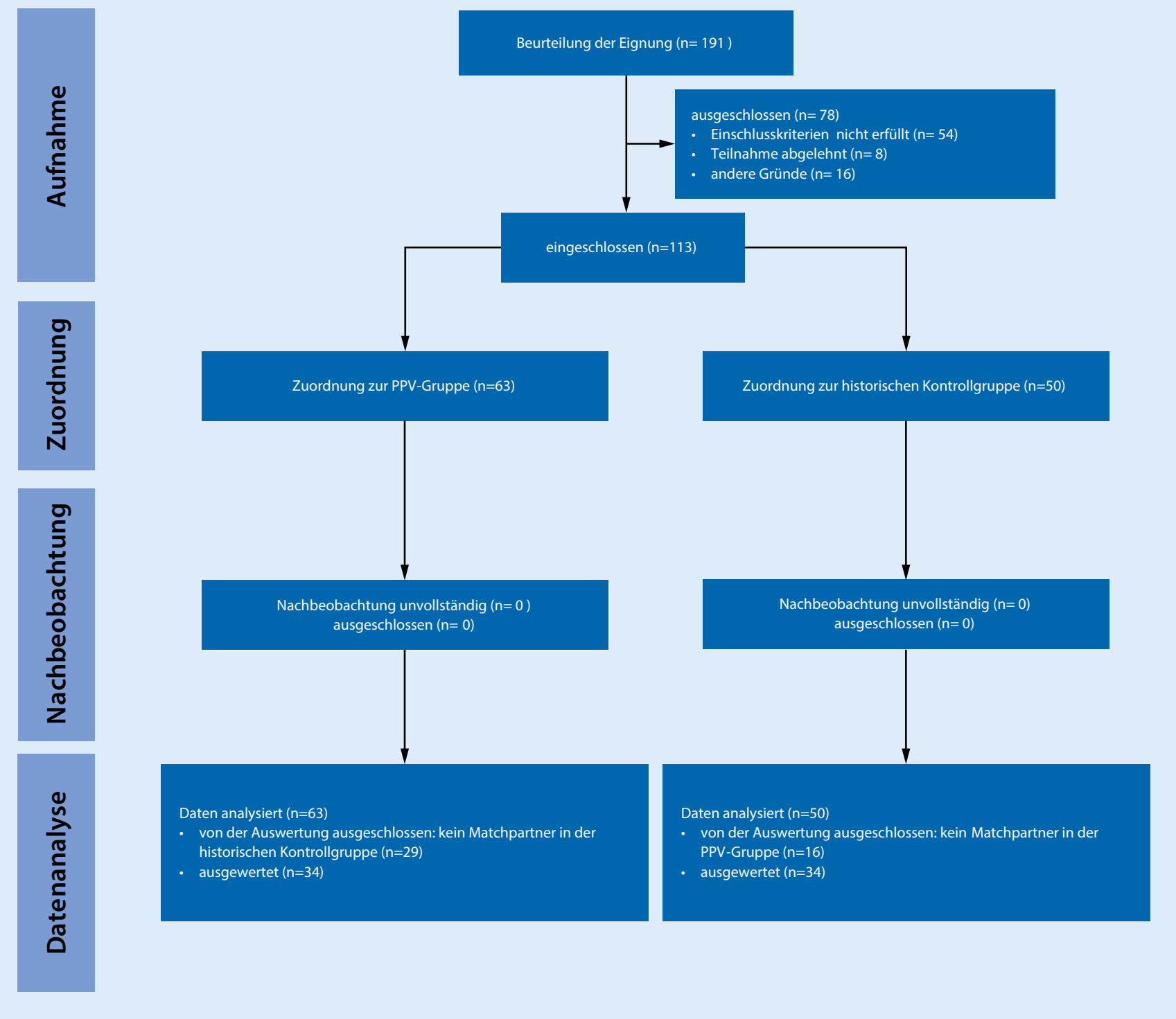

Abb. 1 A Flussdiagramm zur PPV-Gruppe und historischer Kontrollgruppe im Rahmen der Evaluation eines perioperativen Maßnahmenbündels bei Knietotalendoprothese. PPV patient*innenzentrierte perioperative Versorgung

tientenschulung, modifiziertes chirurgisches und anästhesiologisches Vorgehen, nichtinvasive Schmerztherapie, frühzeitige Mobilisation) bei Knietotalendoprothese verglichen.

\section{Material und Untersuchungs- methoden}

Nach schriftlicher Genehmigung des nach den STROBE-Leitlinien [30] erstellten Studienprotokolls durch die zuständige Ethikkommission (Ethikkommission der Landesärztekammer Rheinland-Pfalz, Vorsitzender: Univ.Prof. Dr. med. habil. Dipl.-Ing. Stephan
Letzel, Bearbeitungsnummer 837.280.18 (10599) vom 07.11.2016), ausführlicher Aufklärung und schriftlicher Einwilligung wurden 34 Patient*innen mit fortgeschrittener radiologisch nachgewiesener Arthrose des Kniegelenks nach ausreichender konservativer Therapie, deutlich eingeschränkter Lebensqualität und Indikation zur Implantation einer Knietotalendoprothese (Knie-TEP) nach Einführung des Maßnahmenbündels patient ${ }^{\star}$ innenzentrierte perioperative Versorgung (PPV) der Universitätsmedizin Mainz in einer prospektiven, monozentrischen Observationsstudie untersucht (PPV-Gruppe) und mit „matched-
pair"-Kontrollen (•Infobox 1) einer Vergleichspopulation $(n=34)$ vor Einführung des Maßnahmenbündels verglichen (Kontrollgruppe). Einen Überblick ergibt das Flussdiagramm in • Abb. 1.

Da die Studie nicht prospektiv randomisiert, sondern als prospektive Beobachtungsstudie mit einem historischen Kollektiv als Kontrollgruppe geplant war, wurde auf eine Registrierung bei einer öffentlichen Datenbank für klinische Studien verzichtet. Der primäre Endpunkt (Dauer der Anästhesieeinleitung), aufgrund dessen die Abschätzung der Stichprobengröße erfolgte, war vor Studienbeginn in dem der Ethikkommis- 
Anaesthesist 2021 · 70:213-222 https://doi.org/10.1007/s00101-020-00874-8

(c) Der/die Autor(en) 2020

\section{J. Spielberger · F. Heid · I. Schmidtmann · P. Drees · U. Betz · W. Schwaderlapp · G. Pestel}

\section{Patient*innenzentrierte perioperative Versorgung: perioperative Prozessqualität, Effektivität von Schmerztherapie und Mobilisationsfortschritt nach Implementation eines Maßnahmenbündels bei Knietotalendoprothese}

\section{Zusammenfassung}

Hintergrund. In den USA wurde das Konzept des "perioperative surgical home“ initialisiert, in dem ein teamorientiertes Vorgehen einen umfassenderen und zügigeren Heilverlauf erzielen soll.

Fragestellung. Evaluation des Effekts eines interdisziplinären Maßnahmenbündels (patient*innenzentrierte perioperative Versorgung, PPV) auf Aspekte der Prozessqualität unter deutschen Rahmenbedingungen. Material und Methoden. Nach Einführung des PPV-Maßnahmenbündels (1. Patient ${ }^{*}$ innenseminar, 2 . spezifische Chirurgietechnik, 3. spezifische Anästhesietechnik, 4. Physiotherapiebeginn am Operationstag) wurden 34 Patient*innen mit elektiver Knietotalendoprothese prospektiv untersucht und mit "matched-pair"-Kontrollen verglichen. Endpunkte sind Dauer der Einleitungszeit (primär) und Krankenhausverweildauer, Ruhe- und Belastungsschmerz am 1. postoperativen Tag (numerische Analogskala), und Mobilisationsfortschritt (MBF) an den postoperativen Tagen 1, 3 und 6 (sekundär). Gruppenvergleiche wurden mit WilcoxonMann-Whitney-Tests auf Nichtunterlegenheit durchgeführt. Im Fall von Nichtunterlegenheit wurde anschließend auf Überlegenheit getestet.

Ergebnisse. Die Einleitungszeit in der PPV-Gruppe betrug im Median 13,5 min (Kontrollgruppe: $60 \mathrm{~min}, p<0,0001$ ), die Krankenhausverweildauer betrug in der PPVGruppe 8 Tage (Kontrollgruppe: 12 Tage, $p<0,0001)$. Am ersten postoperativen Tag betrug die mediane Ruheschmerzstärke in der PPV-Gruppe 30 (Kontrollgruppe: 20); die Belastungsschmerzstärke war in beiden Gruppen gleich (Median 40). Die Mobilisation der Patienten*innen der PPV-Gruppe gelang an den postoperativen Tagen 1, 3 und 6 besser (jeweils $p<0,0001$ ).

Schlussfolgerung. Das Konzept der patient*innenzentrierten perioperativen Versorgung (PPV) erscheint vielversprechend genug, um weitere klinische Studien zu rechtfertigen.

Schlüsselwörter

Behandlungspfad $\cdot$ Heilverlauf $\cdot$ Patient ${ }^{*}$ innenzentrierte perioperative Versorgung.PPV

\section{Patient-centered perioperative vigilance: perioperative process quality, effectiveness of pain treatment and mobilization progress after implementation of a treatment bundle for total knee endoprosthesis}

\begin{abstract}
Background. The perioperative surgical home is a patient-centered, team-based model of care developed in the USA to coordinate diagnosis, treatment and followup; however, due to different healthcare systems, scientific findings in the USA cannot be simply transferred to Germany.

Objective. This preliminary study was carried out to evaluate the effects of a new interdisciplinary treatment bundle (patientcentered perioperative vigilance, PPV) in a German university hospital.

Material and methods. After IRB approval and written informed consent, 34 patients (PPV group) undergoing elective endoprosthetic surgery were enrolled after introduction of the PPV bundle (1. preoperative patient
\end{abstract}

education, 2. specific surgical technique, 3. specific anesthesia technique, 4. start of mobilization on day of operation) and compared to historic matched pairs (HMP) for age cohort, ASA-PS, body mass index, and sex. We hypothesized that PPV shortens induction time (primary outcome). Secondary outcomes were length of hospital stay (LOS), resting pain and pain with movement on postoperative day 1 and mobilization progress on postoperative days 1, 3 and 6. Groups were compared with Wilcoxon-Mann-Whitney test for noninferiority. In the case of noninferiority, a Wilcoxon-Whitney-Mann test for superiority was additionally applied.

Results. The median anesthesia induction time was $13.5 \mathrm{~min}$ for PPV and $60 \mathrm{~min}$ for
HMP $(p<0.0001)$. The LOS was 8 days for PPV and 12 days for HMP $(p<0.0001)$. Resting pain on postoperative day 1 was 20 for PPV (30 for HMP). Pain with movement was identical (median 40). Mobilization progress was better for PPV on days 1, 3 and 6 $(p<0.0001$ for each day).

Conclusion. The concept of patient-centered perioperative vigilance (PPV) shortens induction time and hospital length of stay. Mobilization improves with PPV on day 1. Higher pain scores in PPV seem to be clinically insignificant, which warrants further study.

\section{Keywords}

Treatment bundle - Perioperative surgical home $\cdot$ Patient-centered outcomes · PSH sion zur Beschlussfassung vorgelegten Studienprotokoll schriftlich niedergelegt worden.

Geplante Regionalanästhesie, Dauermedikation mit Opioiden, ASA-PS-Klassifikation $>3$, kognitive und/oder kommunikative Defizite, die nach Meinung des Prüfarztes den Erfolg einer adäquaten Studienaufklärung ungewiss erschienen ließen, sowie Teilnahme an pharmakologischen Studien innerhalb der letzten 28 Tage galten als Ausschlusskriterien.

\section{Anteile des Maßnahmenbündels}

Bei einem obligaten Patient ${ }^{*}$ innenseminar [6] 2 bis 4 Wochen vor dem geplanten Operationstermin werden Patient ${ }^{*}$ in und Coach (eine ausgewählte Vertrauensperson) der perioperative Ablauf und Details der Operation, der Anästhesie und der Schmerztherapie sowie der Physiotherapie und daraus resultierender Entlassungskriterien (Ankleiden ohne Hilfe, selbstständiges Ein- und Aussteigen aus dem Bett, selbstständiges Hinsetzen auf und Aufstehen von einem 


\section{Infobox 2 Maßnahmenbündel „Patient*innenzentrierte peri- operative Versorgung, PPV" der Universitätsmedizin Mainz bei Knietotalendoprothese}

- Enge interdisziplinäre Abstimmung in regelmäßigen Treffen der Steuergruppe

- Präoperatives Patient*innenseminar mit Patientencoach

- Organisatorische Optimierung von präoperativen Abläufen

- Balancierte Allgemeinanästhesie ohne Katheterverfahren

- Opioidsparende multimodale Analgesie

- Tranexamsäure intraoperativi.v. und lokal

- Hämoderivate nach PBM-Kriterien

- Atraumatische Operationstechnik und intraoperative Kontrolle des Operationserfolges

- Physiotherapeutische Maßnahmen zur Steigerung der individuellen Handlungsfähigkeit mit Aktivitätsangeboten wie Bewegungsparcours und Outdoor-Gruppe

Stuhl oder von der Toilette, persönliche Hygiene ohne Hilfe, Mobilisierung mit Unterarmgehhilfen, $70 \mathrm{~m}$ Gangdistanz mit Unterarmgehhilfen, ausreichende Schmerzbehandlung mit Schmerzstärke $<50$ auf einer numerischen Analogskala (0: keine Schmerzen; 100: stärkster vorstellbarer Schmerz) bei Aktivität) durch Verantwortliche der jeweiligen Fachbereiche in einer offenen und wertschätzenden Atmosphäre dargestellt.

Die organisatorische Optimierung von präoperativen Abläufen (z.B. chirurgische und anästhesiologische Aufklärung am Tag des Patient ${ }^{\star}$ innenseminars) und zeitnaher Einbestellung der Patient ${ }^{*}$ innen am Operationstag mit kurzer Nüchternheitsphase wurde akribisch geplant und durchgeführt.

Die Allgemeinanästhesie wurde mit Propofol, Sufentanil und Atracurium gewichtsadaptiert eingeleitet und mit Sufentanil und Sevofluran aufrechterhalten. Auf Nervenblockaden und Katheterverfahren zur Analgesie wurde verzichtet. Stattdessen wurde eine opioidsparende multimodale Analgesiestrategie mit oraler Gabe von Dexamethason, Etoricoxib und Oxycodon sowie zusätzlicher Etablierung einer intraoperativen lokalen Infiltrationsanalgesie (LIA, Anlage durch den Operateur) [7] verfolgt (200 ml Ropivacain $0,2 \%$, davon $150 \mathrm{ml}+1 \mathrm{mg}$ Epine- phrin periartikulär in sämtliche chirurgisch adressierten Gewebe sowie $50 \mathrm{ml}$ ohne Epinephrin s.c.). Als Reservemedikation im Aufwachraum bei Schmerzdurchbruch wurde Piritramid vorgehalten. Tranexamsäure wurde bei Operationsbeginn ( $1 \mathrm{~g}$ i.v.) und nach Kapselverschluss (2g i.a.) gegeben [8]. Hämoderivate wurden nach PBM-Kriterien [22] verabreicht.

Patient ${ }^{*}$ innen der Kontrollgruppe erhielten vor Narkoseeinleitung sonographisch gesteuert einen N.-femoralisKatheter. Zur postoperativen kontinuierlichen Katheteranalgesie wurde Ropivacain $0,2 \%$ perineural infundiert $(6-12 \mathrm{ml} / \mathrm{h})$. Ergänzend wurden die Patient ${ }^{*}$ innen mit einer i.v.-PCA (patientenkontrollierte Analgesie) versorgt (Piritramidbolus $3 \mathrm{mg}$, Sperrzeit $10 \mathrm{~min}$, 8-h-Maximum $30 \mathrm{mg}$, keine Basalrate). Begleitend wurde in Abhängigkeit von Vorerkrankungen Diclofenac, Paracetamol oder Metamizol verordnet. Hinsichtlich der Durchführung der Allgemeinanästhesie gab es keine Gruppenunterschiede.

Im Rahmen des Maßnahmenbündels wurde chirurgisch auf eine atraumatische Operationstechnik geachtet, mit Vermeidung von pneumatischer Blutsperre [12], minimaler Schnittführung mit Nutzung natürlicher Muskellücken, Verzicht auf Drainagen und intraoperativer Kontrolle des Operationserfolges mittels bildgebendem Verfahren.

Physiotherapeutisch waren alle Bewegungen und Körperhaltungen erlaubt [11]. Lagerungsmaterial und passive Bewegungsschienen wurden nicht eingesetzt. Im Mittelpunkt standen Maßnahmen zur Steigerung der individuellen Handlungsfähigkeit („empowerment“) und Motivation zu selbstständiger Aktivität, das Erarbeiten der Selbstständigkeit im Alltag, Angehörigenanleitung, Vermittlung von Eigenübungsprogrammen, Vorgaben strukturierter Aktivitätsprogramme und regelmäßiges Screening der Funktionsfähigkeit. Die Patient ${ }^{\star}$ innen verließen bereits am Operationstag aktiv das Bett und wurden ermuntert, Aktivitätsangebote wie Bewegungsparcours und Outdoor-Gruppe in Anspruch zu nehmen.

\section{Endpunkte}

Primärer Endpunkt war die Dauer der Einleitungszeit, welche im Rahmen der Anästhesiedokumentation erhoben wurde. Dieser Parameter wurde aufgrund der Erwartung des stärksten Effektes bei Einführung des PPV-Maßnahmenbündels gewählt. Als sekundäre Endpunkte wurden die Krankenhausverweildauer (LOS) sowie Ruhe- und Belastungsschmerz (RSZ, BSZ) am 1. postoperativen Tag gemessen. Zur Quantifizierung wurde eine numerische Analogskala (0: keine Schmerzen; 100: stärkster vorstellbarer Schmerz) verwendet. Als weiterer sekundärer Endpunkt wurde der Mobilisationsfortschritt (MBF) mithilfe einer Mobilisationsstufenskala von 0 bis 5 (0: keine Mobilisation, 1: Patient ${ }^{\star}$ in sitzt an der Bettkante, 2: Patient*in steht, 3: Patient ${ }^{*}$ in geht auf Zimmerebene, 4: Patient ${ }^{*}$ in geht auf Flurebene, 5: Patient ${ }^{*}$ in steigt Treppen) an den postoperativen Tagen 1, 3 und 6 erhoben.

\section{Statistische Methoden}

Ziel war es, die mittlere Einleitungszeit mit einem 95\%-Konfidenzintervall $\mathrm{zu}$ bestimmen, das eine 95\%-Überdeckungswahrscheinlichkeit hat und einen Bereich \pm 10 min um die beobachtete mittlere Einleitungszeit umfasst. Eine retrospektive Analyse früher dokumentierter Einleitungszeiten ergab annähernd normal verteilte Einleitungszeiten mit einer Standardabweichung von 23,6 min. Mit dieser Grundlage ergibt sich für die PPV-Gruppe ein Stichprobenumfang von $n=34$.

Zum Vergleich der Patient*in der PPV-Gruppen mit den historischen Kontrollen wurden für die primären und sekundären Zielgrößen WilcoxonMann-Whitney-Tests angewandt, da eine Normalverteilung nicht überall angenommen werden konnte. Zunächst wurde jeweils geprüft, ob gegenüber der historischen Kontrolle Nichtunterlegenheit nachgewiesen werden konnte. Die Nullhypothese besagte jeweils, dass $P(X>Y)+\frac{1}{2} P(X=Y) \leq \frac{1}{2}-\epsilon$, mit Nichtunterlegenheitsschranke $\epsilon=0,1$. Dabei steht $\mathrm{X}$ für den in der Vergleichsgruppe beobachteten Wert der 
Tab. 1 Demografische Daten und Matching von PPV-Gruppe und historischer Kontrollgruppe

Patientenkollektiv

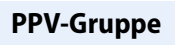

\begin{tabular}{|c|c|c|c|c|c|c|}
\hline \multirow{6}{*}{ Alter (Jahre) } & \multicolumn{2}{|c|}{ PPV-Gruppe } & \multicolumn{2}{|c|}{ Kontrollgruppe } & \multirow{5}{*}{ Tota } & \\
\hline & $n$ & $M W \pm S D$ & $n$ & $M W \pm S D$ & & \\
\hline & 34 & $67,5 \pm 11,1$ & 34 & $66,9 \pm 12,6$ & & \\
\hline & \multicolumn{4}{|c|}{ Patientenkollektiv } & & \\
\hline & \multicolumn{2}{|c|}{ PPV-Gruppe } & \multicolumn{2}{|c|}{ Kontrollgruppe } & & \\
\hline & $n$ & $\%$ & $n$ & $\%$ & $n$ & $\%$ \\
\hline \multicolumn{7}{|l|}{ Geschlecht } \\
\hline - Weiblich & 18 & 53 & 18 & 53 & 36 & 53 \\
\hline - Männlich & 16 & 47 & 16 & 47 & 32 & 47 \\
\hline \multicolumn{7}{|l|}{ Gewichtsklasse } \\
\hline$-\mathrm{BMl}<25 \mathrm{~kg} / \mathrm{m}^{2}$ & 4 & 12 & 4 & 12 & 8 & 12 \\
\hline - BMI 25-30 & 12 & 35 & 12 & 35 & 24 & 35 \\
\hline$-\mathrm{BMI}>30$ & 18 & 53 & 18 & 53 & 36 & 53 \\
\hline \multicolumn{7}{|l|}{ ASA-PS } \\
\hline-1 & 2 & 6 & 2 & 6 & 4 & 6 \\
\hline-2 & 19 & 56 & 19 & 56 & 38 & 56 \\
\hline-3 & 13 & 38 & 13 & 38 & 26 & 38 \\
\hline
\end{tabular}

ASA-PS American Society of Anesthesiologists Physical Score, BMI Body-Mass-Index, MW Mittelwert, $P P V$ patient*innenzentrierte perioperative Versorgung, $S D$ Standardabweichung

Zielgröße, Y für den in der PPV-Gruppe beobachteten Wert, und höhere Werte werden als ungünstig angesehen. Wenn dies möglich war, wurde anschließend noch auf Überlegenheit geprüft. Alle Tests wurden einseitig zum 2,5\%-Niveau durchgeführt. Eine Korrektur für multiples Testen wurde nicht vorgenommen; es wird also nur das lokale Signifikanzniveau eingehalten.

Die Analysen wurden mit SAS 9.4 (SAS Institute, Cary, NC, USA) und $\mathrm{R}$ 3.5.0 (R Foundation for Statistical Computing, Wien, Österreich) durchgeführt.

\section{Ergebnisse}

34 Patient*innen wurden prospektiv nach Einführung des perioperativen Maßnahmenbündels „patient ${ }^{\star}$ innenzentrierte perioperative Versorgung" untersucht (PPV-Gruppe) und mit 34 Patient $^{*}$ innen vor Einführung des Maßnahmenbündels nach „matchedpair"-Kriterien verglichen. Eine Übersicht der demografischen Daten und des Matching zeigt $\bullet$ Tab. 1.

Bei den historischen Kontrollen betrugt die mediane Einleitungszeit $60 \mathrm{~min}$ (Mittelwert = 61,1 min), im PPV-Kollektiv betrugen Median und Mittelwert je- weils 13,5 min (• Abb. 2). Das 95\%-Konfidenzintervall für den Mittelwert ergab [12,0 min; 15,4 min]. Im Vergleich mit den historischen Kontrollen ergab sich eine signifikant kürzere Einleitungszeit $(p<0,0001)$.

Während die mediane Krankenhausverweildauer ( $\bullet$ Abb. 3) der historischen Kontrollen bei 12 Tagen (IQR 11 bis 14 Tage) lag, war sie bei den PPVPatient*innen mit 8 Tagen im Median (IQR 7 bis 8 Tage) signifikant kürzer $(p<0,0001)$.

Am ersten postoperativen Tag wurde der von den Patient*innen im PPVKollektiv auf einer Skala von 0 bis 100 eine mediane Ruheschmerzstärke von 30 (IQR 2,5-40) angegeben, während die Patient ${ }^{*}$ innen aus der historischen Kontrolle im Median 20 (IQR 10-30) angaben. Die Belastungsschmerzstärke war im Median in beiden Gruppen 40 (IQR PPV 30-60, Kontrollen 30-50). Weder für Ruheschmerz noch für Belastungsschmerz konnte bei PPV Nichtunterlegenheit nachgewiesen werden (Ruheschmerz: $p=0,1711$, Belastungsschmerz $p=0,2512)$ (•Abb. 4).

Eine Übersicht über den Mobilisationsfortschritt gibt • Abb. 5: Am ersten Tag unterschied sich die Mobilisa- tion sehr deutlich zwischen den beiden Patientengruppen $(p<0,0001)$. Während von den Kontrollen nur 9 überhaupt das Bett verließen und standen, verließen alle PPV-Patient ${ }^{*}$ innen das Bett. 18 gingen auf Flurebene, 8 stiegen Treppen. Auch am dritten Tag unterschieden sich die beiden Gruppen noch sehr deutlich in der Mobilisation $(p<0,0001)$. Treppensteigen gelang in der PPV-Gruppe 20 Patient ${ }^{*}$ innen (Kontrollgruppe 0 ). Der Unterschied in der Mobilisation bestand auch noch am sechsten Tag $(p<0,0001)$. Zwar konnten in der Kontrollgruppe zu diesem Zeitpunkt bis auf 2 alle gehen, jedoch nur je 11 auf dem Flur oder bereits Treppen steigen. In der PPV-Gruppe konnten 2 Patient ${ }^{\star}$ innen auf dem Flur gehen; die übrigen verbliebenen 21 dokumentierten Patient*innen konnten Treppen steigen; 8 waren zu diesem Zeitpunkt bereits entlassen.

\section{Diskussion}

Patient ${ }^{*}$ innenzentrierte perioperative Versorgung (PPV) ist ein interdisziplinäres Maßnahmenbündel der Universitätsmedizin Mainz bei Knietotalendoprothese. Es besteht aus obligater präoperativer Patientenschulung, spezifischer chirurgischer Technik, spezifischer Anästhesietechnik mit Ersatz von Katheteranalgesie durch eine Kombination aus lokaler intraoperativer Analgesie mit opioidsparender multimodaler Analgesie und Beginn der präoperativ eingeübten Physiotherapie (Gehen an Unterarmgehstützen sowie ein Eigenübungsprogramm zur Thromboseprophylaxe, Aktivierung und Dehnung der Beinmuskulatur sowie der Mobilisation der Kniegelenke) noch am Operationstag. PPV bewirkt neben einer deutlich verkürzten Anästhesieeinleitungszeit eine Reduktion der Krankenhausverweildauer sowie einen verbesserten postoperativen Mobilisationsfortschritt unter suffizienter Schmerztherapie.

Die Implementation des Maßnahmenbündels (u. a. Ersatz von Katheteranalgesie durch eine Kombination aus lokaler Intraoperativer Analgesie mit opioidsparender multimodaler Analgesie) führte zu einer Reduktion der Einleitungszeit von im Median $60 \mathrm{~min}$ 


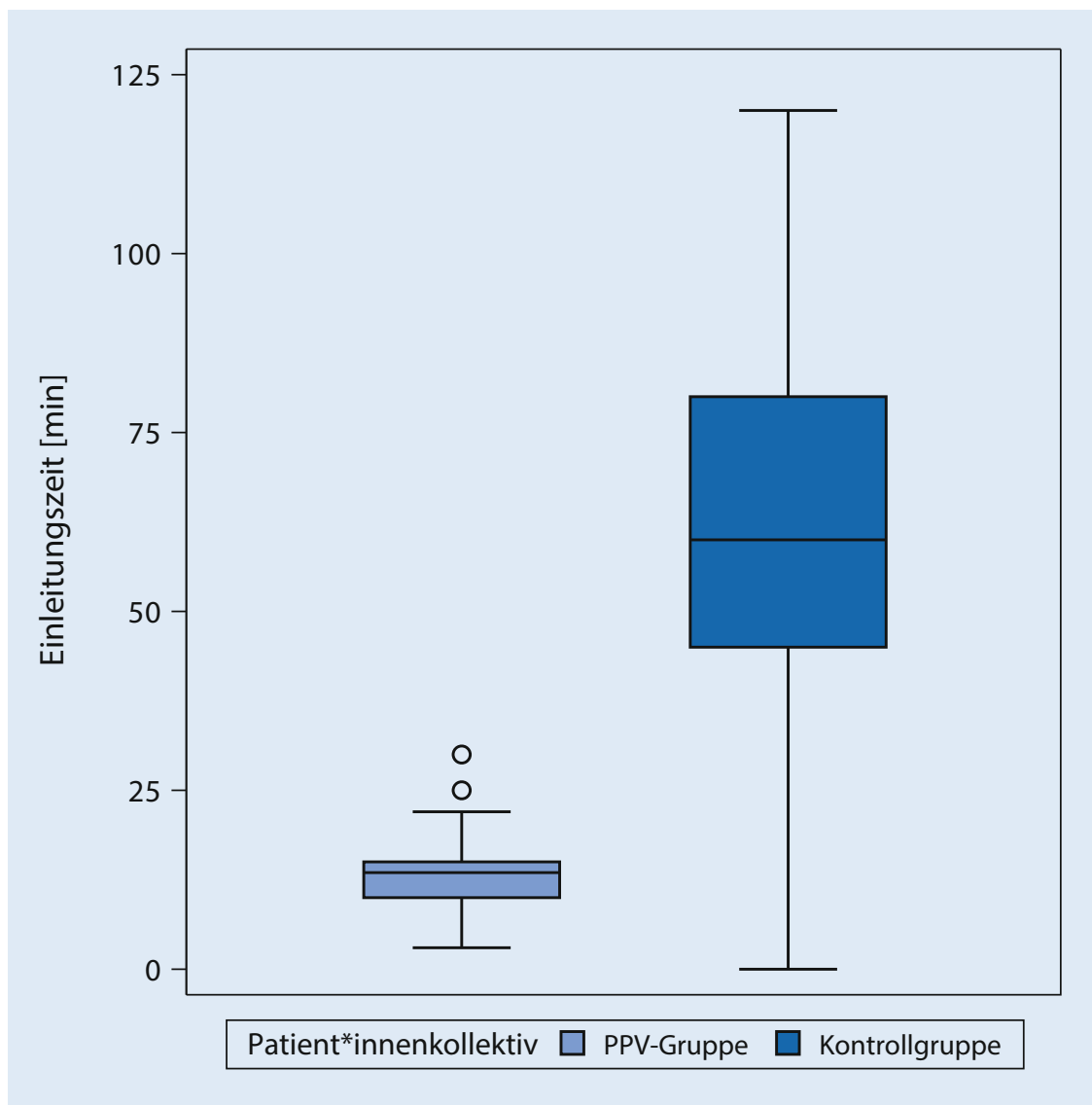

Abb. 2 ॥ Verteilung der Einleitungszeit bei der PPV-Gruppe $(n=34)$ und der historischen Kontrollgruppe ( $n=34)$. Wilcoxon-Test auf Überlegenheit einseitig: $p<0,0001$. PPV patient*innenzentrierte perioperative Versorgung

auf $13,5 \mathrm{~min}$. Eine Zeiteinsparung von mehr als $45 \mathrm{~min}$ im Rahmen der Einleitung gestattet nicht nur einen zügigeren Operationsbeginn im Tagesablauf, sondern eröffnet auch Gestaltungsräume für die Verwendung von Anästhesiepersonal durch Verminderung der Intensität bei der Personalbindung. Im USamerikanischen Schrifttum wurde eine Effizienzsteigerung der perioperativen Prozesse als Möglichkeit postuliert, eine „durchschlagende Innovation“ [10] im Gesundheitswesen mit dem Ziel der einfacheren, zugänglicheren und preisgünstigeren Gesundheitsversorgung zu erreichen. In einer monozentrischen Observationsstudie, bei der ein Zweijahreszeitraum vor Einführung eines perioperativen Maßnahmenbündels in der Endoprothetik mit einem Zweijahreszeitraum nach Einführung des Maßnahmenbündels verglichen worden war, zeigte sich eine signifikante Zunahme des Anteils von Fällen mit pünktlichem
(Median) um 4 Tage entsprechende Aufmerksamkeit.

In 2 US-amerikanischen Studien [18, 29] konnte durch die Einführung eines spezifischen Behandlungsprotokolls keine Veränderung der Krankenhausverweildauer erreicht werden. Allerdings wurde in keiner der beiden Studien die Einführung eines therapeutischen Maßnahmenbündels evaluiert. Generell ist in den USA gemäß einer landesweiten Erhebung an über 1 Mio. Patient ${ }^{*}$ innen die Krankenhausverweildauer bei endoprothetischen Eingriffen mit 3 Tagen (Median) sehr kurz, bemerkenswert erscheint dabei aber, dass eine Entlassung aus dem Krankenhaus (Verlegung nach Hause) nur in 47,1 \% der Fälle beschrieben wird [4].

Einer ausführlichen präoperativen Aufklärung und Schulung bei $\mathrm{Pa}$ tient ${ }^{*}$ innen, die sich einer Knietotalendoprothesenoperation unterziehen, wird ein hoher Stellenwert zur positiven Beeinflussung des Heilverlaufs eingeräumt [16]. In einer nordenglischen Studie an insgesamt 472 Patient $^{*}$ innen konnte durch ein vor der Operation interdisziplinär durchgeführtes Programm zu Aufklärung und Schulung eine Verminderung der Krankenhausverweildauer (Median) von 7 Tagen auf 5 Tage erreicht werden [14]. Möglicherweise trägt die in unserem Maßnahmenbündel integrierte obligate Patientenschulung zur beobachteten Verminderung der Krankenhausverweildauer bei.

Nach Implementation des Maßnahmenbündels bei elektiven Knietotalendoprothesen wurde bei betroffenen Patient ${ }^{*}$ innen am ersten postoperativen Tag im Median eine Schmerzintensität von 30 (2,5-40) auf einer numerischen Analogskala von 0-100 gemessen, gegenüber einer Schmerzintensität von 20 (10-30) vor Einführung des Maßnahmenbündels. Hinsichtlich des Belastungsschmerzes am ersten postoperativen Tag wurden im Median Werte von 40 (30-60) nach Einführung des Maßnahmenbündels erhoben, gegenüber Werten von 40 (30-50) vor Einführung des Maßnahmenbündels. Zwar bewegt sich anscheinend die erlebte Schmerzintensität der Patient*innen nach Einführung des Maßnahmenbündels noch im tolerablen 


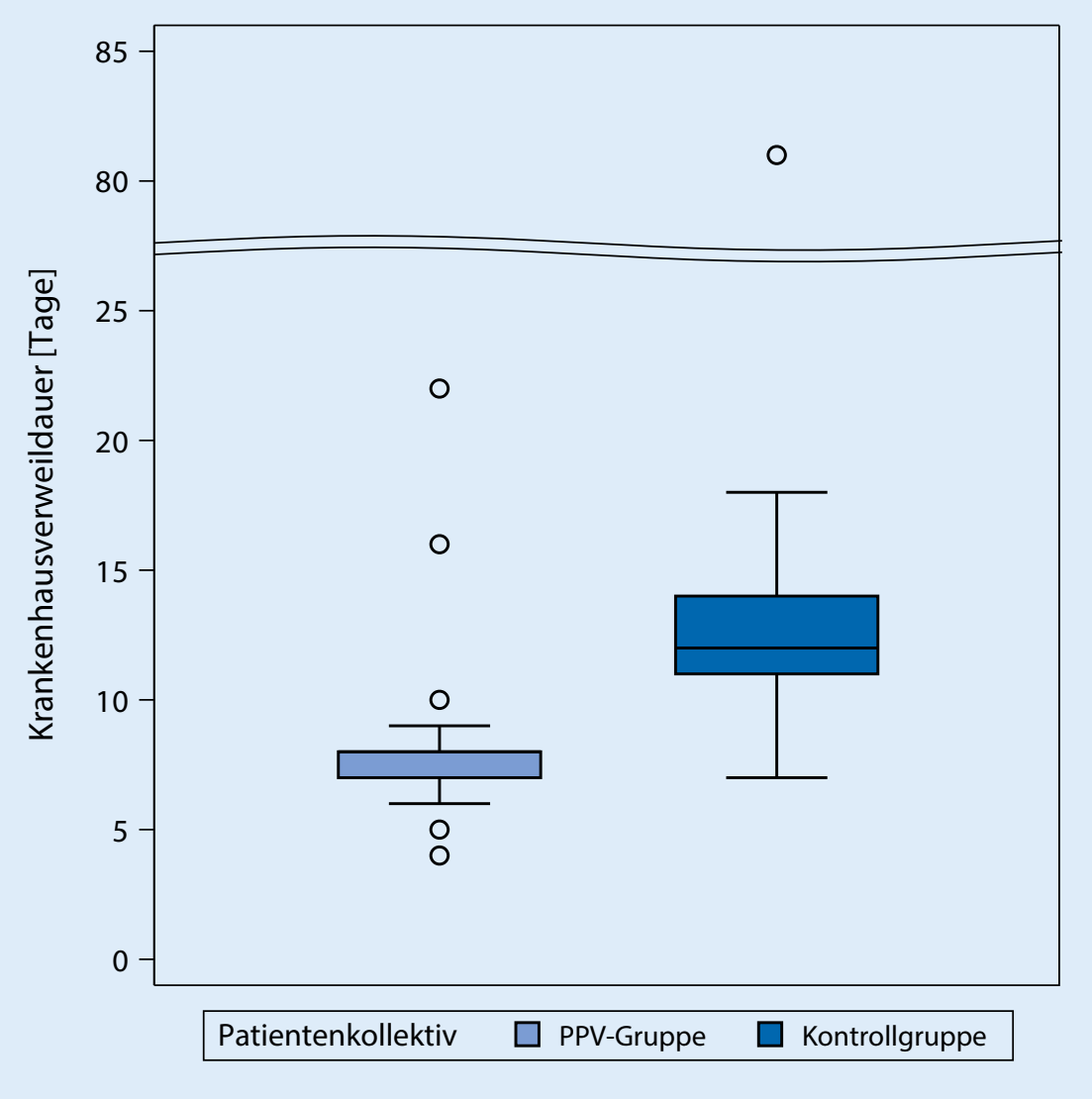

Abb. 3 A Verteilung der Krankenhausverweildauer, einschließlich Operationstag, bei der PPV-Gruppe $(n=34)$ und der historischen Kontrollgruppe $(n=34)$. Wilcoxon-Test auf Überlegenheit einseitig: $p<0,0001$. PPV patient*innenzentrierte perioperative Versorgung

Bereich; ein statistischer Nachweis der Nichtunterlegenheit des schmerztherapeutischen Bemühens nach Einführung des Maßnahmenbündels gelang jedoch nicht.

Drei US-amerikanische deskriptive Beobachtungsstudien $[3,9,26]$ nach Einführung eines „Perioperative-surgical-home“-Konzepts berichten über Schmerzintensität am 1. postoperativen Tag; eine Differenzierung zwischen Ruheschmerz und Belastungsschmerz wurde nicht vorgenommen. Die in vorliegender Untersuchung beobachtete postoperative Schmerzintensität ist somit vergleichbar mit den Befunden der US-amerikanischen Studien. Allerdings ist aufgrund des Vergleichs mit der Kontrollgruppe (vor Einführung des Maßnahmenbündels) zukünftig der Fragestellung Vermeidung von Schmerzen/ Schmerztherapie bei Patient*innen mit Knietotalendoprothese unter Durchführung des Maßnahmenbündels besonde- und ein Drittel gerade in den Stand $\mathrm{zu}$ mobilisieren. Gründe waren die allgemein akzeptierte Vorstellung, dass Patient*innen nach der Operation zunächst ruhen sollten; Übelkeit, Schmerz und Schwindel waren schnell akzeptierte Gründe gegen aktive Mobilisierung, d.h., Mobilität wurde weniger unterstützt und eingefordert. Zudem bestanden zahlreiche Mobilitätsbarrieren (Lagerungsschienen, Blasenkatheter, Femoraliskatheter, i.v.-Zugänge, Bewegungsverbote). Die Einführung des Maßnahmenbündels bewirkte eine hochsignifikante Steigerung der postoperativen Mobilisierbarkeit. Der Erfolg der Frühmobilisierung in vorliegender Untersuchung erscheint vergleichbar mit einer amerikanischen Studie an 146 Patient ${ }^{*}$ innen nach endoprothetischem Eingriff [9], bei der von $100 \%$ iger Mobilisierung in den Stand innerhalb von $24 \mathrm{~h}$ nach Operation berichtet wird. Inwieweit die Mobilisierbarkeit nach Kniegelenkersatz durch Maßnahmen der „Prähabilitation“ (d.h. Maßnahmen VOR einem operativen Eingriff, mit dem Ziel der präoperativen Stärkung zur beschleunigten postoperativen Erholung) gesteigert werden kann, ist augenblicklich Gegenstand intensiver klinischer Forschung. Während eine frühere Metaanalyse bei Patient*innen mit endoprothetischem Gelenkersatz einen nur bescheidenen Effekt der Prähabilitation auf den Heilverlauf vermutete [32], konnte eine Metaanalyse bei solchen mit Kniegelenkersatz Verbesserungen der Mobilisierbarkeit mit Auswirkungen auf die Krankenhausverweildauer nachweisen [17]. Eine zusätzliche Steigerung des Effektes von Prähabilitationsmaßnahmen könnte zukünftig durch eine patientenspezifische Therapie im Sinne von "personalisierter Medizin“ erreicht werden [2].

Die vorliegende Arbeit beinhaltet mehrere Limitationen: Erstens handelt es sich nicht um eine randomisierte prospektive Studie. Zwar wurden $\mathrm{Pa}-$ tient ${ }^{*}$ innen nach Einführung des Maßnahmenbündels prospektiv untersucht, bei der Vergleichsgruppe handelt es sich aber über eine retrospektiv ausgewählte Kontrolle nach "matched-pair"-Kriterien. Ein solcher Vergleich ist methodenbedingt mit Unzulänglichkeiten behaftet, 


\section{Originalien}
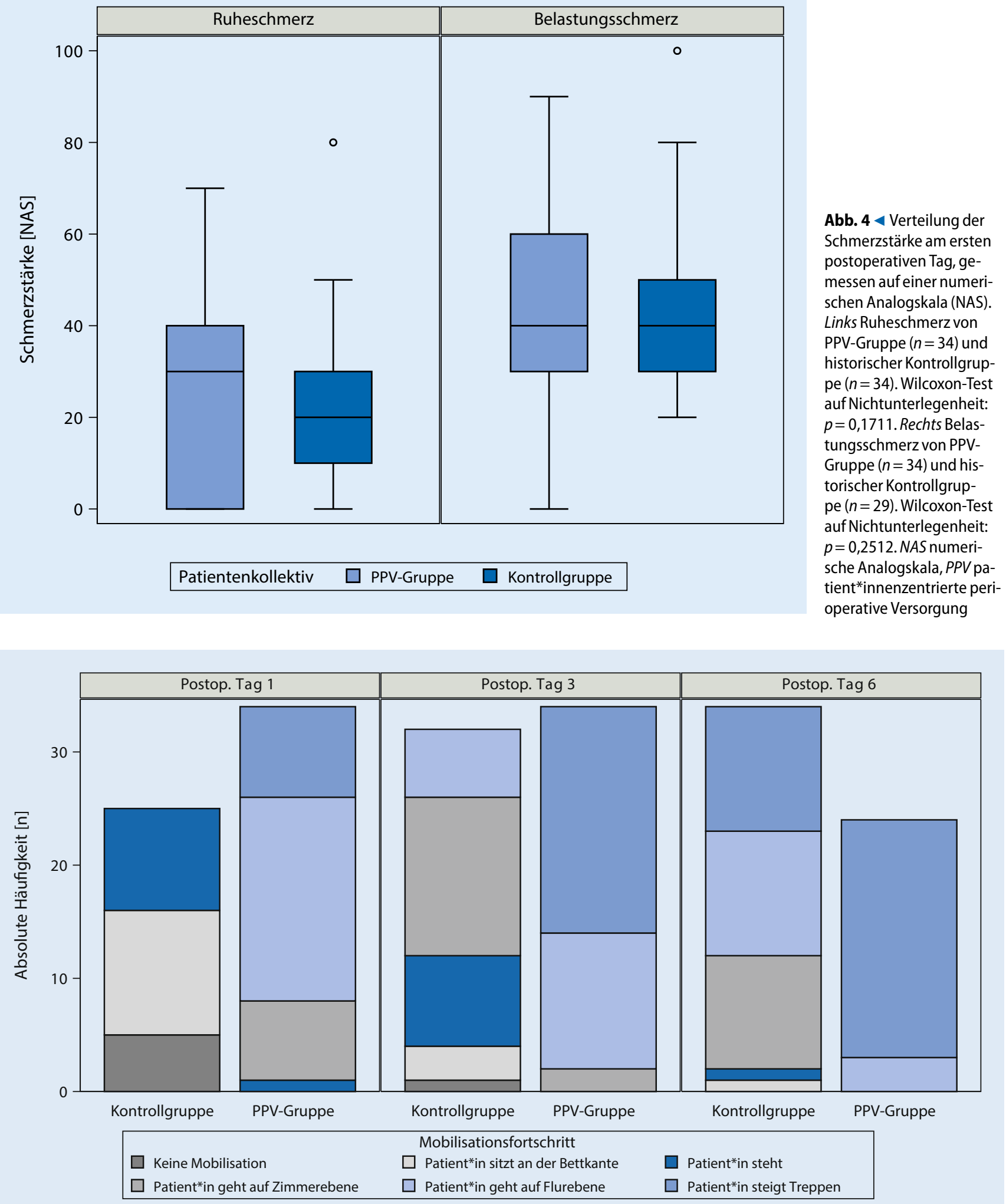

Abb. $5 \Delta$ Mobilisationsfortschritt an den postoperativen Tagen 1, 3 und 6 bei der PPV-Gruppe (Tag 1:n=34, Tag 3: $n=34$, Tag 6: $n=24)$ und der historischen Kontrollgruppe (Tag 1: $n=25$, Tag 3: $n=34$, Tag 6: $n=34$ ). Wilcoxon-Test auf Überlegenheit: $p<0,0001$ für alle Tage. Postop. postoperativer, PPV: patient*innenzentrierte perioperative Versorgung 
bietet aber zumindest Anhaltspunkte, die in reinen Beobachtungsstudien ohne Vergleichsgruppe nicht möglich sind. Zweitens erscheint der Mobilisationsfortschritt in der prospektiven Gruppe gegenüber der historischen Kontrolle augenfällig und besitzt statistische Signifikanz. Aufgrund der unterschiedlichen Fallzahlen an den postoperativen Tagen 1, 3 und 6 wären definitive Schlussfolgerungen derzeit allerdings verfrüht. Drittens kann die Generalisierbarkeit der gefundenen Ergebnisse hinterfragt werden, da es sich bei der Universitätsmedizin Mainz um ein Krankenhaus der Maximalversorgung handelt, mit entsprechenden Auswirkungen auf das Patientenkollektiv und klinikinterne Handlungsabläufe. Schließlich haben alle Schlussfolgerungen aufgrund der insgesamt geringen Gruppengrößen eher deskriptiven als beweisenden Charakter.

Zusammenfassend wurden in einer prospektiv angelegten Beobachtungsstudie an 34 Patient $^{*}$ innen mit elektiver Knietotalendoprothese nach Einführung eines interdisziplinären Maßnahmenbündels Effekte der Prozessqualität untersucht und einem „matchedpair,-Vergleich (Alter, Geschlecht, BMIGruppe, ASA-PS-Klasse) einer historischen Kohorte unterzogen. Dabei zeigte sich eine deutliche Verkürzung von Anästhesieeinleitungszeit und Krankenhausverweildauer bei verbesserter Mobilisierbarkeit. Die Ergebnisse weisen darauf hin, dass die Einführung und konsequente Anwendung eines interdisziplinar vereinbarten Maßnahmenbündels im Sinne des US-amerikanischen „perioperative surgical home“-Konzepts auch unter den Bedingungen des deutschen Gesundheitswesens vorteilhafte Effekte für Patient ${ }^{*}$ innen, Behandelnde und Gesellschaft haben kann. Vor diesem Hintergrund haben Einrichtungen der Universitätsmedizin Mainz und Kooperationspartner das durch den Innovationsfonds beim Gemeinsamen Bundesausschuss geförderte PROMISEProjekt mit dem Ziel der Entwicklung eines Best-Practice-Leitfadens für einen optimierten Gesamtversorgungsprozess bei Gelenkerkrankungen gestartet. Die Autor*innen schlagen als deutschen
Begriff „patient*innenzentrierte perioperative Versorgung (PPV)“ vor.

\section{Kernaussagen}

- Das interdisziplinäre therapeutische Maßnahmenbündel „patient*innenzentrierte perioperative Versorgung, PPV“ der Universitätsmedizin Mainz führt für die Patient ${ }^{*}$ innen $\mathrm{zu}$ einem zügigen Heilverlauf.

- „Patient*innenzentrierte perioperative Versorgung, PPV“ ist ein Modell für einen optimierten Gesamtversorgungsprozess bei Patient ${ }^{\star}$ innen mit Knietotalendoprothese.

- „Patient*innenzentrierte perioperative Versorgung, PPV“ beschleunigt die Anästhesieeinleitung und den Mobilitätsfortschritt und verkürzt die Krankenhausverweildauer.

- Die erlebte Schmerzintensität der Patient ${ }^{*}$ innen nach Einführung von „patient*innenzentrierter perioperativer Versorgung, $\mathrm{PPV}^{\text {“ }}$ bleibt im tolerablen Bereich.

\section{Fazit für die Praxis}

- Das Konzept „patient*innenzentrierte perioperative Versorgung, PPV" ist ein interdisziplinärer Ansatz auf dem Fundament des Vertrauensgrundsatzes und der strikten Arbeitsteilung aller beteiligten Berufsgruppen mit dem Ziel des optimierten Heilverlaufs für die Patient*innen. Leitmotiv ist die Verzahnung aller patientenrelevanten Tätigkeiten. Die akribische Vorbereitung des Gesamtkonzeptes und dessen Weiterentwicklung im laufenden Betrieb erfordern von allen beteiligten Berufsgruppen konsensorientierte Innovations- und Dialogbereitschaft.

- Das Hinterfragen und Verwerfen vertrauter Automatismen zugunsten von evidenzbasiertem Handeln bedarf kritischer Reflexion und Überzeugungsarbeit, zunächst in den einzelnen Berufsgruppen, dann zwischen den einzelnen Berufsgruppen. Die Benennung von Irrwegen und Sackgassen ist unvermeidlich und unproblematisch, wenn notwendig gewordene Kurskorrekturen auf das gemeinsame Ziel des optimierten Heilverlaufs für die Patient*innen ausgerichtet sind.

- Eine präzise, verständliche und umfassende Patientenschulung ist für die aktive Mitarbeit der Patient*innen unerlässlich.

- Die Steuerung des Gesamtkonzepts, dessen Fortschreibung nach Überprüfen der Zielerreichung und die Aufnahme von Vorschlägen aus der Schwarmintelligenz aller Mitarbeitenden wird durch Verantwortliche, die in regelmäßigem Austausch miteinander stehen, bewerkstelligt.

Korrespondenzadresse
G. Dr. med. habil.
Glinik für Anästhesiologie,
Universitätsmedizin Mainz
Langenbeckstr. 1,
55131 Mainz, Deutschland
pestel@uni-mainz.de

Funding. Open Access funding enabled and organized by Projekt DEAL.

\section{Einhaltung ethischer Richtlinien}

Interessenkonflikt. J. Spielberger, F. Heid, I. Schmidtmann, P. Drees, U. Betz, W. Schwaderlapp und G. Pestel geben an, dass kein Interessenkonflikt besteht.

Alle beschriebenen Untersuchungen am Menschen oder an menschlichem Gewebe wurden mit Zustimmung der zuständigen Ethikkommission, im Einklang mit nationalem Recht sowie gemäß der Deklaration von Helsinki von 1975 (in der aktuellen, überarbeiteten Fassung) durchgeführt. Von allen beteiligten Patient*innen liegt eine Einverständniserklärung vor.

Open Access. Dieser Artikel wird unter der Creative Commons Namensnennung 4.0 International Lizenz veröffentlicht, welche die Nutzung, Vervielfältigung, Bearbeitung, Verbreitung und Wiedergabe in jeglichem Medium und Format erlaubt, sofern Sie den/die ursprünglichen Autor(en) und die Quelle ordnungsgemäß nennen, einen Link zur Creative Commons Lizenz beifügen und angeben, ob Änderungen vorgenommen wurden.

Die in diesem Artikel enthaltenen Bilder und sonstiges Drittmaterial unterliegen ebenfalls der genannten Creative Commons Lizenz, sofern sich aus der Abbildungslegende nichts anderes ergibt. Sofern das betreffende Material nicht unter der genannten Creative Commons Lizenz steht und die betreffende Handlung nicht nach gesetzlichen Vorschriften erlaubt ist, ist für die oben aufgeführten Weiterverwendungen des Materials die Einwilligung des jeweiligen Rechteinhabers einzuholen. 
Weitere Details zur Lizenz entnehmen Sie bitte der Lizenzinformation auf http://creativecommons.org/ licenses/by/4.0/deed.de.

\section{Literatur}

1. American Society of Anesthesiologists (2020) Perioperative surgical home (PSH). https://www. asahq.org/psh.Zugegriffen: 23. Apr. 2020

2. Bandholm T, Wainwright TW, Kehlet H (2018) Rehabilitation strategies for optimisation of functional recovery after major joint replacement. J Exp Orthop. https://doi.org/10.1186/s40634018-0156-2

3. Cyriac J, Garson L, Schwarzkopf R, Ahn K Rinehart J, Vakharia S, Cannesson M, Kain Z (2016) Total joint replacement perioperative surgical home program: 2-year follow-up. Anesth Analg 123:51-62

4. Dexter F, Epstein RH, Sun EC, Lubarsky DA, Dexter EU (2017) Readmissions to different hospitals after common surgical procedures and consequences for implementation of perioperative surgical home programs. Anesth Analg 125:943-951

5. Dexter F, Wachtel RE (2014) Strategies for net cost reductions with the expanded role and expertise of anesthesiologists in the perioperative surgical home. Anesth Analg 118:1062-1071

6. Edwards PK, Mears SC, Lowry Barnes C (2017) Preoperative education for hip and knee replacement: never stop learning. Curr Rev Musculoskelet Med 10:356-364

7. Fan L, Zhu C, Zan P, Yu X, Liu J, Sun Q, Li G (2015) The comparison of local infiltration analgesia with peripheral nerve block following total knee arthroplasty (TKA): a systematic review with metaanalysis. J Arthroplasty 30:1664-1671

8. Franchini M, Mengoli C, Marietta M, Marano G, Vaglio S, Pupella S, Mannucci PM, Liumbruno GM (2018) Safety of intravenous tranexamic acid in patients undergoing major orthopaedic surgery: a meta-analysis of randomised controlled trials. Blood Transfus 16:36-43

9. Garson L, Schwarzkopf R, Vakharia S, Alexander B, Stead S, Cannesson M, Kain Z (2014) Implementation of a total joint replacement-focused perioperative surgical home: a management case report. Anesth Analg 118:1081-1089

10. Goeddel LA, Porterfield JR Jr, Hall JD, Vetter TR (2015) Ethical opportunities with the perioperative surgical home: disruptive innovation, patientcentered care, shared decision making, health literacy, and futility of care. Anesth Analg 120:1158-1162

11. Gromov K, Troelsen A, Modaddes M, Rolfson O, Furnes $\mathrm{O}$, Hallan G, Eskelinen A, Neuvonen P, Husted H (2019) Varying but reduced use of postoperative mobilization restrictions after primary total hip arthroplasty in Nordic countries: a questionnairebased study. Acta Orthop 90:143-147

12. Guler $O$, Mahirogullari $M$, Isyar M, Piskin A, Yalcin S, Mutlu S, Sahin B (2016) Comparison of quadriceps muscle volume after unilateral total knee arthroplasty with and without tourniquet use. Knee Surg Sports Traumatol Arthrosc 24:2595-2605

13. Institut für Qualitätssicherung und Transparenz im Gesundheitswesen (2018) Bundesauswertung zum Erfassungsjahr 2017: Knieendoprothesenversorgung. https://iqtig.org/downloads/
auswertung/2017/kep/QSKH KEP 2017 BUAW V02_2018-08-01.pdf.Zugegriffen:28. Mai 2020

14. Jones S, Alnaib M, Kokkinakis M, Wilkinson M, St Clair Gibson A, Kader D (2011) Preoperative patient education reduces length of stay after knee joint arthroplasty. Ann RColl Surg Engl 93:71-75

15. Kain ZN, Vakharia S, Garson L, Engwall S, Schwarzkopf R, Gupta R, Cannesson M (2014) The perioperative surgical home as a future perioperative practice model. Anesth Analg 118:1126-1130

16. Kash BA, Zhang Y, Cline KM, Menser T, Miller TR (2014) The perioperative surgical home (PSH): a comprehensive review of US and non-US studies shows predominantly positive quality and cost outcomes. Milbank Q 92:796-821

17. Moyer R, lkertK, Long K, Marsh J (2017) The value of preoperative exercise and education for patients undergoing total hip and knee arthroplasty: a systematic review and meta-analysis. JBJS Rev 5:e2

18. Mudumbai SC, Walters TL, Howard SK, Kim TE, Lochbaum GM, Memtsoudis SG, Kain ZN, Kou A, King R, Mariano ER (2016) The Perioperative Surgical Home model facilitates change implementation in anesthetic technique within a clinical pathway for total knee arthroplasty. Healthc (Amst) 4:334-339

19. OECD (2010) Durchschnittliche Krankenhausverweildauer. In: Gesundheit auf einen Blick 2009: OECD-Indikatoren. OECD Publishing, Paris https://doi.org/10.1787/soc_glance-2009-41-de

20. Römer R, Komann M, Weinmann C, Meißner W (2020) Schmerz nach Knietotalendoprothese. Ist die lokale Infiltrationsanästhesie die beste Therapieoption? Schmerz 34:33-40

21. Shepperd S, Iliffe S, Doll HA, Clarke MJ, Kalra L, Wilson AD, Gonçalves-Bradley DC (2016) Admission avoidance hospital at home. Cochrane Database Syst Rev 9:CD7491

22. Society for the Advancement of Blood Management (2019) Webpräsenz. http://www.sabm.org. Zugegriffen:5.Okt. 2019

23. Soffin EM, Gibbons MM, Ko CY, Kates SL, Wick E, Cannesson M, Scott MJ, Wu CL (2019) Evidence review conducted for the agency for healthcare research and quality safety program for improving surgical care and recovery: focus on anesthesiology for total knee arthroplasty. Anesth Analg 128:441-453

24. Statistisches Bundesamt (2018) Fallpauschalenbezogene Krankenhaus-statistik (DRG-Statistik): Operationen und Prozeduren der vollstationären Patientinnen und Patienten in Krankenhäusern

25. Statistisches Bundesamt (2017) Diagnosedaten der Patienten und Patientinnen in Krankenhäusern (einschl. Sterbe-und Stundenfälle)

26. Tse BK, Walters TL, Howard SK, Kim TE, Memtsoudis SG, Sun EC, Kou A, Graham L, King R, Mariano ER (2017) A matched case-control comparison of hospital costs and outcomes for knee replacement patients admitted postoperatively to acute care versus rehabilitation. J Anesth 31:785-788

27. Vetter TR, Ivankova NV, Goeddel LA, McGwin G Jr, Pittet JF (2013) An analysis of methodologies that can be used to validate if a perioperative surgical home improves the patient-centeredness, evidence-based practice, quality, safety, and value of patient care. Anesthesiology 119:1261-1274

28. Vetter TR, Boudreaux AM, Jones KA, Hunter JM Jr, Pittet JF (2014) The perioperative surgical home: how anesthesiology can collaboratively achieve and leverage the triple aim in health care. Anesth Analg 118:1131-1136
29. Vetter TR, Barman J, Hunter JM Jr, Jones KA, Pittet JF (2017) The effect of implementation of preoperative and postoperative care elements of a perioperative surgical home model on outcomes in patients undergoing hip arthroplasty or knee arthroplasty. Anesth Analg 124:1450-1458

30. Von Elm E, Altman DG, Egger M, Pocock SJ, Gøtzsche PC, Vandenbroucke JP (2008) Das Strengthening the Reporting of Observational Studies in Epidemiology (STROBE-) Statement. Leitlinien für das Berichten von Beobachtungsstu dien. Internist (Berl) 49:688-693

31. Wainwright TW, Gill M, McDonald DA, Middleton RG, Reed M, Sahota O, Yates P, Ljungqvist O (2020) Consensus statement for perioperative care in total hip replacement and total knee replacement surgery: Enhanced Recovery After Surgery $\left(\right.$ ERAS $^{\circledR}$ ) Society recommendations. Acta Orthop 91:3-19

32. Wang L, Lee M, Zhang Z, Moodie J, Cheng D, Martin J (2016) Does preoperative rehabilitation for patients planning to undergo joint replacement surgery improve outcomes? A systematic review and meta-analysis of randomised controlled trials. BMJ Open 6:e9857 